\title{
GAMMA-RAY IMAGING FOR EXPLOSIVES DETECTION
}

\author{
G.A. de Nolfo*a , S. D. Hunter*b , L.M. Barbier ${ }^{\mathrm{b}}$, J.T. Link ${ }^{\mathrm{c}}$, S. Son ${ }^{\mathrm{a}}$,

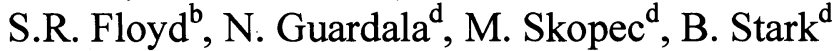 \\ ${ }^{a}$ NASA/Goddard Space Flight Center, Code 661, Greenbelt, MD, USA 20771; \\ ${ }^{\mathrm{b}} \mathrm{UMBC} / \mathrm{CRESST} / \mathrm{NASA}$, Code 661, Greenbelt, MD USA 20771; \\ ${ }^{\mathrm{c} U S R A / C R E S S T / N A S A}$, Code 661, Greenbelt, MD USA 20771; \\ ${ }^{\mathrm{d}}$ Naval Surface Warfare Center, Carderock, MD USA 20814
}

\begin{abstract}
We describe a gamma-ray imaging camera (GIC) for active interrogation of explosives being developed by NASA/GSFC and NSWC/Carderock. The GIC is based on the Three-dimensional Track Imager (3-DTI) technology developed at GSFC for gamma-ray astrophysics. The 3-DTI, a large volume time-projection chamber, provides accurate, $\sim 0.4 \mathrm{~mm}$ resolution, 3-D tracking of charged particles. The incident direction of gamma rays, $\mathrm{E}_{\gamma}>6 \mathrm{MeV}$, are reconstructed from the momenta and energies of the electron-positron pair resulting from interactions in the 3-DTI volume. The optimization of the 3-DTI technology for this specific application and the performance of the GIC from laboratory tests is presented.
\end{abstract}

Keywords: Micro-well detector (MWD), Gamma-ray Imaging Camera (GIC), WGPu, HEU

\section{INTRODUCTION}

The Department of Defense has been tasked to develop means of standoff interrogation to detect small quantities of WGPu, HEU, and high explosives (HE). WGPu is readily detected passively by its fast neutron emission (Hunter et al. these proceedings). HEU can also be detected using neutron imaging. HEU detection requires active interrogation with neutrons to increase the low, natural neutron emission rate by induced fission. Detection of HE can also be achieved by employing active interrogation with neutrons, however, the most promising method requires imaging gamma rays from thermal neutron capture on nitrogen, ${ }^{14} \mathrm{~N}(\mathrm{n}, \gamma){ }^{15} \mathrm{~N}^{*}$. De-excitation of the ${ }^{15} \mathrm{~N}^{*}$ results in several gamma-ray lines with energies from 4.48 to $10.82 \mathrm{MeV}$ (Kinsey et al., 1950). Fifteen percent of the de-excitations result in the release of a $10.82 \mathrm{MeV}$ gamma ray (Mernagh, et al. 1977).

Measurement of the gamma rays for imaging a source of HE is based on their dominant interactions in matter, Compton scattering and pair production. For both processes one detects and measures the tracks of resulting recoil electron or electron-positron pair. Our gamma-ray imaging technology, developed for astrophysics in the $5 \mathrm{MeV}$ to $50 \mathrm{GeV}$ energy range, not only can be used here, but can be optimized for the detection of the gamma rays resulting from neutron capture on nitrogen.

The Gamma-ray Imaging Camera (GIC) is based on the NASA/GSFC Three-Dimensional Track Imager (3-DTI) detector technology being developed for medium- and high-energy gamma-ray telescopes. The 3-DTI, a large volume time-projection chamber, provides accurate and precise $(\sim 0.4 \mathrm{~mm}$ resolution) three-dimensional tracking of charged particles.

The GIC is sensitive to gamma rays that interact via Compton scattering or pair production. The relative strengths of these two channels is a strong function of energy. Restricting ourselves to pair production and its characteristic ' $\mathrm{V}$ ' formed by the electron-positron pair results in a detection mode with properties of strong background rejection. Thus, the 3-DTI detector for GIC has been optimized as a gamma-ray pair telescope, i.e., to measure the momentum of the electron-positron pair. The incident directions and energies of the gamma rays are reconstructed from these momentum measurements. 
We describe the development of a $10 \times 10 \times 15 \mathrm{~cm}^{3}$ GIC prototype detector, Figure 1 , and subsequent tests in the laboratory using radioactive sources. The goal of the GIC design is to provide a large cubic meter, high-resolution camera, with full $360^{\circ}$ azimuth sensitivity, that is capable of passively detecting $\mathrm{HE}$ at reasonable stand-off distances. The development of GIC will take place in three stages, starting with the already built and tested $10 \times 10 \times 15 \mathrm{~cm}^{3}$ prototype, followed by a scale up $50 \times 50 \times 30 \mathrm{~cm}^{3}$ prototype, and finally a $1 \mathrm{~m}^{3} \mathrm{GIC}$ camera that will be ready for realistic platforms.

\section{TECHNICAL DESCRIPTION}

The underlying principle of the Gamma-ray Imaging Camera (GIC) is to measure the energy and position in three dimensions of charged particles traversing the camera medium (xenon gas mixture at $3 \mathrm{~atm}$ ). Gamma-rays, entering the active volume, interact with the detector gas via Compton scattering and pair production. While GIC is capable of identifying Compton-scatter events, particularly in the low background environments expected from naturally occurring high-energy gamma radiation, we focus in this paper on the identification of pair-production events. At high energies $(E>30 \mathrm{MeV})$ pair production dominates, although a significant number of pair events will be observed down to lower energies. The resulting electron and positron leave a trail of ionization as they traverse the active volume. The characteristic vertex formed from pair production serves to identify incoming gamma rays from other charged particle interactions. The incident direction and energy of the incoming photon is determined by accurately measuring the momenta and energies of the electron and positron. Precise measurements of the kinematics of the incoming gamma rays provides a powerful tool for source identification and for minimizing background.

GIC is comprised of a large volume gas time projection chamber (TPC) with two-dimensional, gas micro-well detector (MWD) readout, see Fig. 1. The TPC is filled with a xenon gas mixture that acts as a large volume, low density, homogenous tracker. Incident high-energy gamma-rays enter the TPC and interact via Compton scattering and pair production. For energies greater than $\sim 30 \mathrm{MeV}$, pair production dominates in xenon and the resulting electron and positron travel through the chamber leaving an ionization trail along their trajectory. The TPC measures the position of the charge particle track and the energy loss by ionization in the gas: It is bounded at the top with a drift electrode and an array of MWDs at the bottom. A cage of electric-field shaping biased wires defines the sides of the active volume and provides a uniform drift field $(\sim 900 \mathrm{~V} / \mathrm{cm})$. The ionization charge along the tracks drifts toward the MWDs at the bottom. The MWDs form a two-dimensional array of gas proportional counters. The MWDs are orthogonal electrode strips rigidly affixed to an insulating substrate. The wells themselves are defined by holes in the cathode strips and in the insulators that expose the orthogonal anode strips below. The micro-wells are maintained at a bias voltage such that the electric field is $10^{4}-10^{5} \mathrm{~V} / \mathrm{cm}$. Thus, the

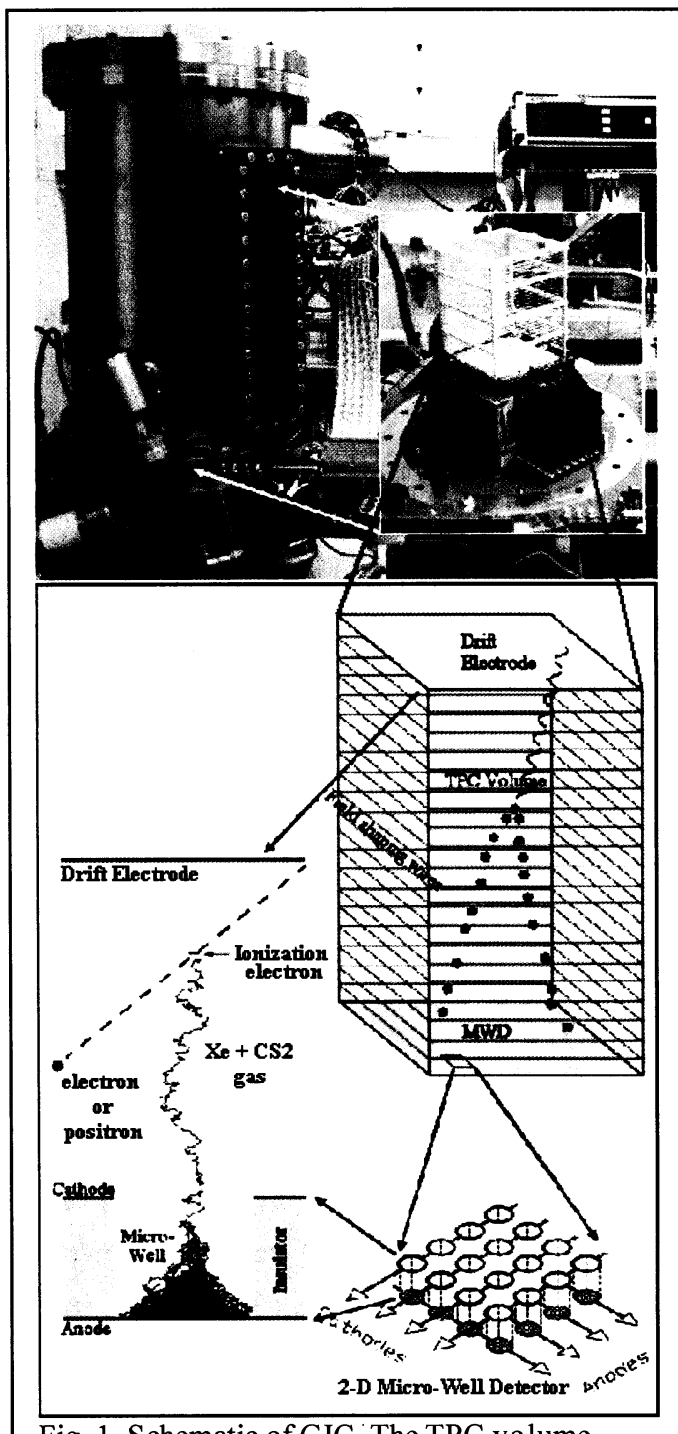

Fig. 1. Schematic of GIC. The TPC volume, MWD, and micro-well operation. The TPC drift field is defined by the cathode plane and the drift electrode. An energetic charged particle traversing the TPC produces ionization electrons that drift into the micro-wells, where, in the intense electric field, they produce an avalanche. The avalanche charge is collected on the anode and an equal and opposite charge is collected on the cathode.

charge, as it enters the wells, produces an ionization avalanche. The electrons are collected on the anode while equal and opposite mirror charges are set up on the cathodes. The orthogonal strips, thus, provide 2D imaging. 
To achieve 3-D reconstruction of a track, the $z$-coordinate of the ionization charge is determined by recording the time structure of the avalanche charge signals on each anode and cathode. The drift velocity of the ionization charge in the gas determines the translation from time to spatial coordinate. The drift velocity of the ionization charge (free electrons) in xenon is on the order of $30 \mathrm{~mm} / \mu \mathrm{s}$, but depends strongly on additive gases $\left(\mathrm{CH}_{4}, \mathrm{CO}_{2}, \mathrm{CH}_{3} \mathrm{NO}_{2}\right.$, etc.) and drift field (Piesart \& Sauli, 1984). Furthermore, because this velocity is significantly higher than the thermal velocity of the gas, electron drift has significant longitudinal and transverse diffusion that smears out the spatial structure of the ionization charge (track structure) after only a few $\mathrm{cm}$ of drift. The drift velocity of the ionization charge can be substantially reduced by the addition of gaseous $\mathrm{CS}_{2}$, a molecule with moderate electron affinity that efficiently scavenges the ionization electrons forming negative anions. The anions drift in thermal equilibrium with the gas and their drift velocity, $\sim 0.1 \mathrm{~mm} / \mu \mathrm{s}$, is about $10^{-3}$ of that for free electrons. Drifting in thermal equilibrium also substantially reduces the transverse and longitudinal diffusion (Martoff et al. 2000), greatly increasing the maximum drift distance. These anions drift towards the anodes where, in the strong electric field of the micro-well, the electrons are stripped off and an electron cascade is produced in the well. In addition, the slow drift velocity reduces the required sampling rate of the transient digitizers that record the arrival time of the ionization charge.

The signals from each anode and cathode are processed though front-end electronics and a transient digitizer. Each FEE consists of a charge sensitive pre-amplifier and a pulse shaper with gain. The charge avalanche in a well induces the same and opposite polarity signal and both signals are readout through an anode and a cathode, respectively. The

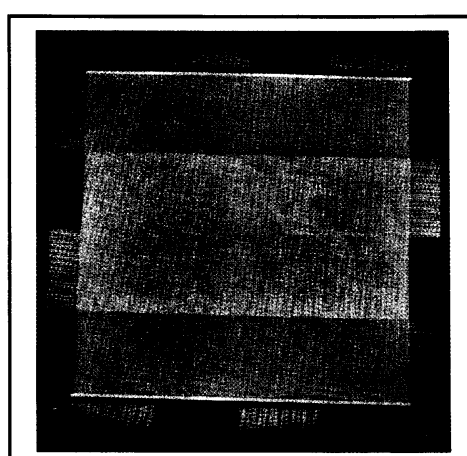

Fig. 2. Micro-well detector. Transient Digitizer (TD) consists of a differential receiver, 12-bit ADC, and a common control FPGA. The TD operates at a sampling rate of $2.5 \mathrm{Msamples} / \mathrm{s}$ (i.e., the FEE is read out every $400 \mathrm{~ns}$ ). After the analogue signal is digitized, the value is stored in a circular buffer with a size of 32,000 Samples/channel. Events, satisfying a trigger threshold, are captured by a PC via USB interface.

\section{MANUFACTURING AND PERFRMANCE CHARACTERISTICS}

\subsection{MWD Manufacturing and Performance}

We have developed an in-house UV laser ablation technique for fabrication of $5 \times 5 \mathrm{~cm}^{2}$ MWDs (Deines-Jones 2002b) and demonstrated stable proportional operation at gas gains up to $3 \times 10^{4}$, spatial resolution of $\sim 200 \mu \mathrm{m}$ (Black et al. 2000,

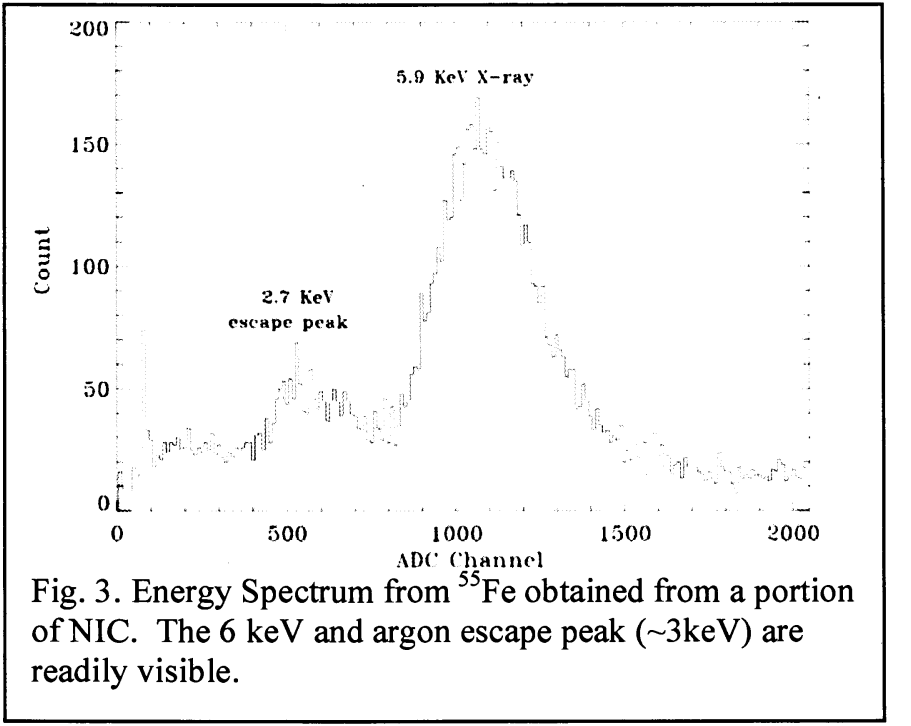

Deines-Jones et al. 2002a), and FWHM energy resolution of $10 \%$ at $20 \mathrm{keV}$ in $\mathrm{P}-10(90 \% \mathrm{Ar} / 10 \%$ $\mathrm{CH}_{4}$ ) and $18 \%$ resolution at $6 \mathrm{keV}$ in $95 \% \mathrm{Xe} / 5 \% \mathrm{CO}_{2}$. We are currently setting up our laboratory for the continue in-house fabrication of MWDs in order to fine-tune the fabrication process.

We have been working with several commercial vendors to develop viable fabrication processes that can be scaled to $50 \times 50 \mathrm{~cm}^{2}$ areas (Hunter, 2006). MWDs with $10 \times 10 \mathrm{~cm}^{2}$ areas have been produced and tested, see Figure 2. The micro-wells are $200 \mu \mathrm{m}$ in diameter and $200 \mu \mathrm{m}$ deep, on $400 \mu \mathrm{m} \times 400 \mu \mathrm{m}$ center-tocenter pitch. We have integrated these MWDs into a $10 \times 10 \times 15 \mathrm{~cm}^{3}$ GIC prototype. We demonstrate an energy resolution of $\sim 18 \%$ using an ${ }^{55} \mathrm{Fe}$ source in the lab with $\mathrm{P}-10 / \mathrm{CS}_{2}$. Figure 3 shows the ${ }^{55} \mathrm{Fe}$ spectrum obtained with the $5.9 \mathrm{keV} \mathrm{x}$-ray peak and the argon escape peak at $\sim 3 \mathrm{keV}$.

\subsection{GIC "Proof of Principle" Performance}

Characterization of GIC has been achieved using a "Proof of Principle" detector (Figure 4) that incorporates $5 \times 5 \times 9$ $\mathrm{cm}^{3}$ MWDs. This detector configuration, sized to fit in a $6^{\prime \prime}(15 \mathrm{~cm})$ diameter vacuum chamber, supports a MWD and 


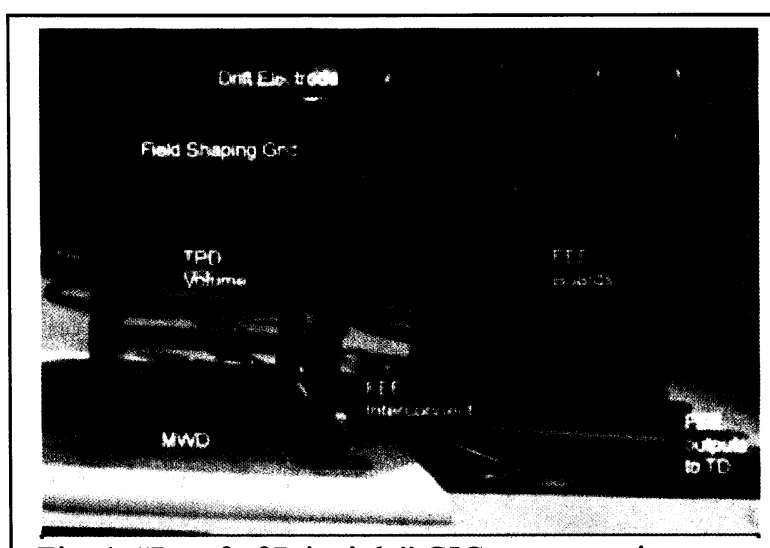

Fig. 4. "Proof of Principle" GIC prototype in laboratory. drift electrode with 2.5 or $9 \mathrm{~cm}$ drift distance, and interface to 96 channels of FEE. A vacuum pump and multi-gas manifold allows this chamber to be easily evacuated and filled with a variety of gas mixtures up to $3 \mathrm{~atm}$.

Using the proof-of-principle 3-DTI detector, we have demonstrated three-dimensional track imaging of electrons from gamma-ray interactions in a variety of gas mixtures: P10 , helium, and xenon, with $\mathrm{CS}_{2}$. We have measured an energy resolution $(\triangle E / E)$ of $\sim 20 \%$ at $5.9 \mathrm{keV}$ in $\mathrm{P}-10 / \mathrm{CS}_{2}$ at a gas gain of $10^{3}$. Good angular resolution of a pair tracker such as GIC is a trade off between the number of samples along the track and the effects of Coulomb scattering. Essentially, one must make many measurements of the track within the first few milli-radiation lengths (milli-RL), before multiple Coulomb scattering begins to dominate. The key to improving angular resolution is to reduce the granularity
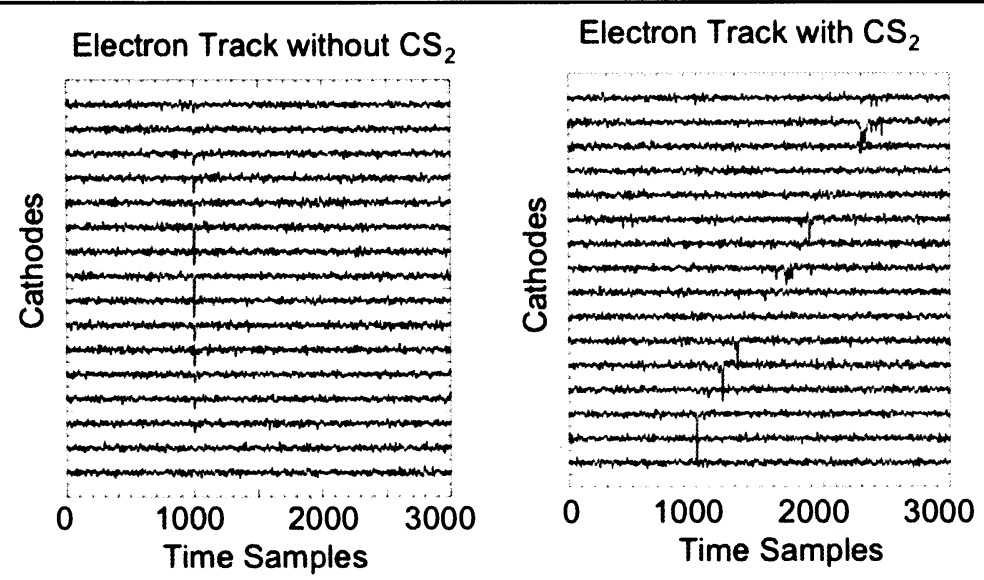

Fig. 5. Electron track (sample time versus chathode) in GIC with (left) and without (right) the addition of $\mathrm{CS}_{2}$.

(number of radiation lengths per sample). The best granularity to date is obtained with emulsion detectors, which have a granularity of typically $3 \times 10^{-5} \mathrm{RL} / \mathrm{sample}$. Another way to significantly improve the granularity is to decrease the density, in other words, use a tracker that has gas as the sample medium. A gas medium improves the granularity by reducing coulomb scattering, minimizing the passive volume (such as converter foils typically used with pair telescopes) and simply increasing the resolution for sampling. The raw TD data from an electron track produced from a ${ }^{90} \mathrm{Sr}$ source is shown in figure 5 . With 400 ns sampling and an $x-y$ pitch of $400 \mu \mathrm{m}$, the granularity of GIC is $8 \times 10^{5} \mathrm{RL} / \mathrm{sample}$, on par with emulsion detectors. The structure visible in the raw TD data, corresponding to the MWD avalanche signals of groups of ionization electrons, illustrates that GIC is capable of recording the track with high resolution including the statistics of the ionization process, energy loss mechanism, and Molière scattering.

The high granularity at a fixed TD sample rate is, in part, possible due to the slow drift times accomplished by the introduction of negative ions, such as $\mathrm{CS}_{2}$. Figures 5 illustrates the difference between an electron track registered in GIC, with and without the addition of $\mathrm{CS}_{2}$, respectively. In addition, since diffusion is significantly reduced by employing negative ion drift, we can expand the active depth of GIC, thus increasing the effective aperture.

\subsection{GIC "Proof of Principle" Imaging}

The raw TD data of the electron-positron pair recorded from a $6.12 \mathrm{MeV}$ gamma ray $\left({ }^{16} \mathrm{O}\right.$ excited state) interaction in $\mathrm{P}$ $1080 \% / \mathrm{CS}_{2} 20 \%$ at a total pressure of $0.55 \mathrm{~atm}$ is shown in Figure 6 . The drift field was $880 \mathrm{~V} / \mathrm{cm}$ and MWD field was $4 \times 10^{4} \mathrm{~V} / \mathrm{cm}$. The apparent truncation of the tracks is due to the particles leaving the active volume. The temporal coincidence of the anode and cathode signals allows the raw TD data to be translated into three spatial coordinates and energy deposition. The $x$ and $y$ coordinates of each coincidence TD pulse are determined by the anode and cathode 


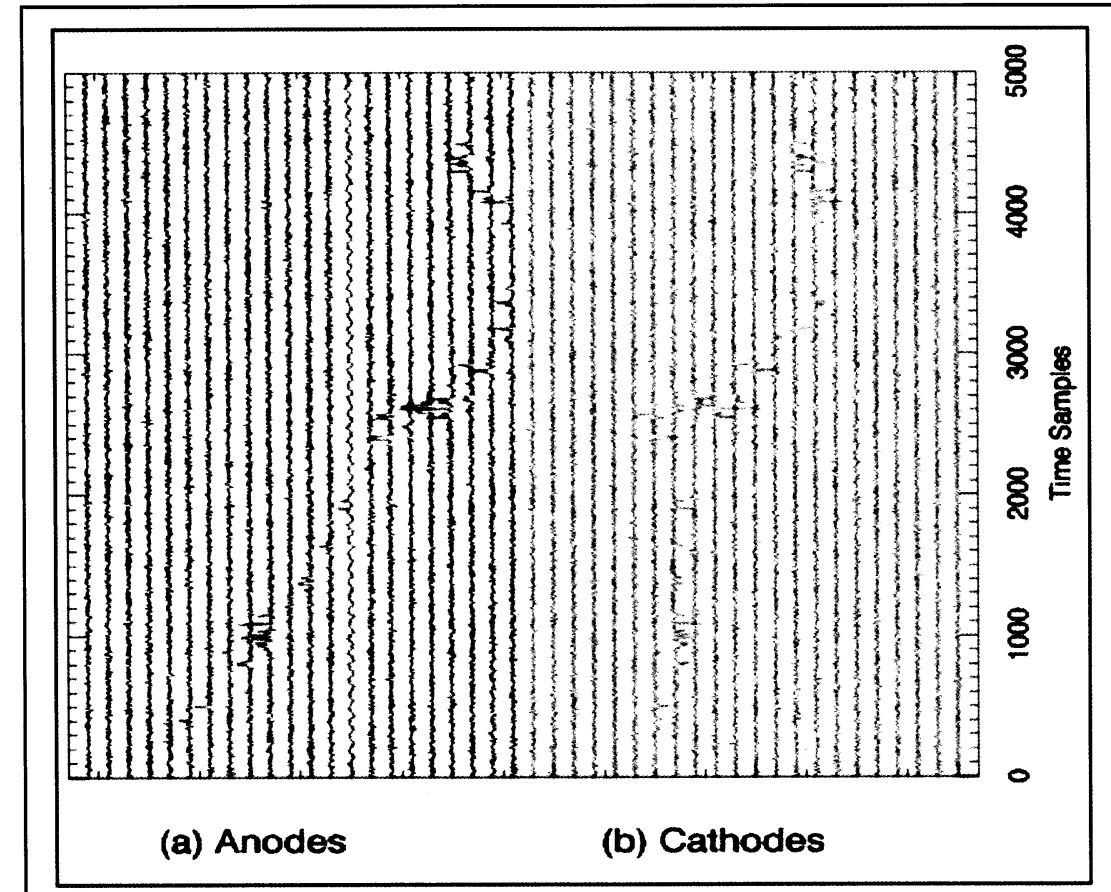

Fig. 6. Raw TD data of a pair production event in GIC. The electron/positron tracks are seen in the anode/cathode versus sample time.

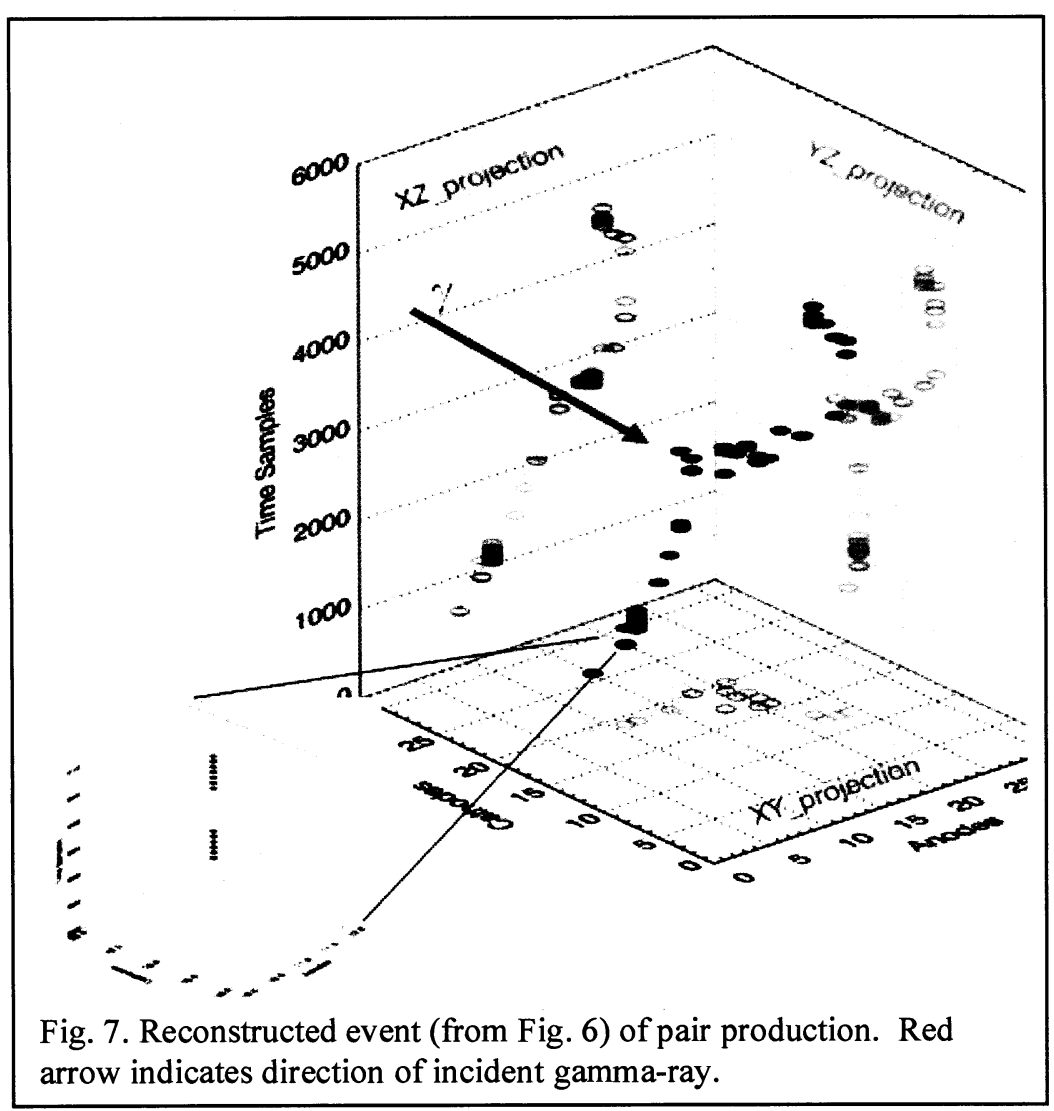




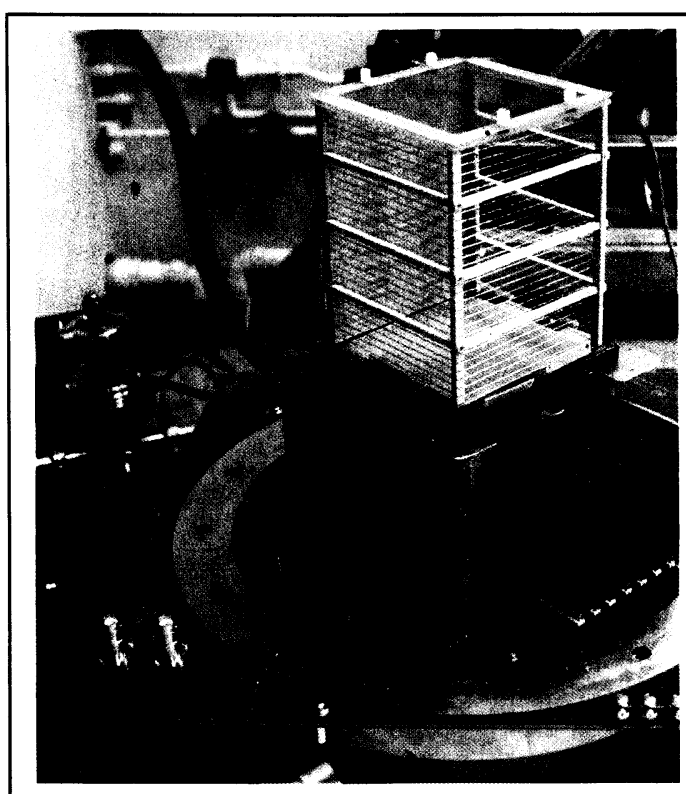

Fig. 8. GIC prototype in laboratory without vacuum chamber.

strip locations. The $\mathrm{z}$ coordinate is derived from the sample time and the negative-ion drift velocity. The signal amplitude is proportional to the energy loss $(\mathrm{dE} / \mathrm{dx})$. We refer to the spatial and energy deposition as the voxel data, $(x, y, z, \Delta E)$.

The 3-D reconstruction of the raw pair image data (Fig. 6) into voxel data is shown in Fig. 7. Complete reconstruction of this event is not possible because the electron and position escaped from the limited active volume. Nevertheless, arrows indicate our best estimate of the electron-positron directions and incident gamma-ray direction. Preliminary analysis, based on a drift velocity of $\sim 50 \mathrm{~m} / \mathrm{s}$, indicates an opening angle of $\sim 40^{\circ}$ for the electron-positron pair, consistent with the opening angle of a $6.1 \mathrm{MeV}$ gamma ray (Borsellino 1953).

\section{$3.410 \times 10 \times 15 \mathrm{~cm}^{3}$ GIC Prototype}

Figure 8 shows our $10 \times 10 \times 15 \mathrm{~cm}^{3}$ prototype GIC in the laboratory without the vacuum chamber. The field-shaping grid rests on top of the MWD. The active volume of the TPC is bounded by this fieldshaping grid. The entire camera is encased within a pressure chamber. The MWD and grid is located above a motherboard, which acts both as a seal for the pressure chamber and connects the detector high voltage and signal cables as well as the FEE low power to the power supplies and TD outside the chamber. The volume of this prototype is large enough to fully contain the majority of gamma-ray interactions, making it possible to fully reconstruct their incident direction and energy. This is possible since the pulse height of the signal registered in the MWD is directly proportional to the energy loss, $\mathrm{dE} / \mathrm{dx}$, along the track. We are currently testing the $10 \times 10 \times 15 \mathrm{~cm}^{3}$ prototype in laboratory with radioactive sources.

\section{SIMULATIONS AND ACCELERATOR TESTS}

We have begun simulations using GEANT 4 to study gamma-ray interactions within GIC. We are currently testing the response uniformity of the MWDs in the laboratory and investigating the gain characteristics of different gas mixtures for the TPC, such as $\mathrm{He} / \mathrm{CS}_{2}$ and $\mathrm{He} / \mathrm{CH}_{3} \mathrm{NO}_{2}$. Complete characterization of the energy resolution and angular resolution will be obtained with an accelerator run at the Positive Ion Accelerator Facility (PIAF) at the Naval Surface Warfare Center in Carderock, MD. The tandem ion accelerator is a Pelletron design, produced by NEC, capable of accelerating positive ions with $3 \mathrm{MV}$ maximum acceleration voltage. The accelerator produces a monoenergetic beam of gamma rays between $0.2-15 \mathrm{MeV}$ and neutrons with energies up to $8 \mathrm{MeV}$.

\section{SUMMARY}

We have successfully demonstrated the capability of imaging high-energy gamma-rays in 3-D with our prototype GIC camera. Imaging gamma-rays is essential for identifying signatures gamma-ray lines from the de-excitation of the ${ }^{15} \mathrm{~N}^{*}$ and thus locating HE. Our upcoming accelerator tests will help to further characterize the angular and energy resolution in addition to refine our image reconstruction analysis.

\section{REFERENCES}

[1] Black, J.K., et al., Proc. SPIE, 4140, 313-323 (2000).

[2] Borsellino, A., Phys. Rev., 89, 1023-12025 (1953).

[3] Deines-Jones, P. et al., NIM-A, 478, 130 (2002a). 
[4] Deines-Jones, P. t al., NIM-A, 477, 55 (2002b).

[5] Hunter, S.D. et al., IEEE, In Publication (2006).

[6] Kinsey Bartholomew, \& Walker, Phys. Rev. 77, 723 (1950).

[7] Martoff, S. et al., NIM-A, 440, 355 (2000).

[8] Mernagh, Harrison, \& McNeill, Phys. Med. Biol., 22, 831 (1977).

[9] Piesart, A. \& Sauli, F., CERN Technical Report, CERN-84-08 (1984). 